\title{
Encephaloduromyosynangiosis (EDMS) on a Pediatric Patient with Cerebral Proliferative Angiopathy
}

\author{
Jingrun $\mathrm{Li}^{1}$, Xinyi Gao ${ }^{2}$, Qian Zhou ${ }^{2}$, Chao Gao ${ }^{2}$, Yujun Liao ${ }^{2}$, Zhenwei Yao ${ }^{2}$ and Bin $\mathrm{Xu}^{1 *}$
}

${ }^{1}$ Department of Neurosurgery, Huashan Hospital of Fudan University, Shanghai, China

${ }^{2}$ Department of Radiology, Huashan Hospital of Fudan University, Shanghai, China

\begin{abstract}
Cerebral proliferative angiopathy (CPA) is a rare cerebral vascular lesion characterized by diffuse vascular abnormalities with intermingled normal brain parenchyma. Cerebral proliferative angiopathy has a complex clinical presentation, and many patients present with ischemic symptoms.

We described a case of a 6 year old boy presenting with transient dizziness with weakness of left limbs. MRI showed the presence of a diffusely dilated extensive vascular lesion mainly located in the corpus callosum, right frontal and temporal lobes. Digital subtraction angiography (DSA) revealed a large hypervascular lesion with a diffuse arterial supply. ${ }^{99 m}$ Tc-ECD SPECT cerebral blood flow perfusion image revealed decreased perfusion in right frontal and parietal lobe. A surgical procedure of encephaloduromyosynangiosis (EDMS) was performed on the patient, and the patient exhibited disappearance of TIAs during the follow-up period.

There is no consensus on treatment of cerebral proliferative angiopathy, but the hemodynamic characteristics of CPA were more similar to moyamoya disease in the PWI-MR study. Furthermore, we speculated that increased regional cerebral blood flow perfusion may relieve clinical symptoms and improve prognosis of patient with CPA. Therefore, we performed encephaloduromyosynangiosis (EDMS) on this pediatric CPA patient with ischemic symptoms. ${ }^{99 m}$ TC-ECD SPECT obtained 6 months after operation showed significant improvement of right frontal and parietal blood perfusion. MR perfusion at follow-up period demonstrated the range and blood flow of vascular lesion decreased significantly compared with preoperative MR perfusion. Follow-up angiography studies showed well compensation by profound neovascularization through the superficial temporal artery, and vascular malformation disappeared around the region supplied from the ECA. EDMS procedure may be a reasonable and effective treatment for patients with ischemia associated with CPA.
\end{abstract}

Keywords: Cerebral proliferative angiopathy Encephaloduromyosynangiosis (EDMS); Cerebral ischemia

\section{Introduction}

Cerebral proliferative angiopathy (CPA) is a rare cerebral vascular disease, which is characterized by diffuse vascular abnormalities with intermingled normal brain parenchyma. Cerebral proliferative angiopathy differs from typical arteriovenous malformations (AVMs) in its angio-morphology, histology, epidemiology, natural history and clinical presentation. Cerebral proliferative angiopathy has a complex clinical presentation, and patients commonly present with epileptic manifestations, headaches, and ischemic neurological deficits [1]. There is no consensus on treatment of cerebral proliferative angiopathy. We report one pediatric cerebral proliferative angiopathy case with cerebral ischemia successfully treated by Encephaloduromyosynangiosis (EDMS).

\section{Case Report}

A 6 year old boy complained of transient dizziness with weakness of left limbs visited an outpatient clinic in Huashan Hospital of Fudan University in November 2015. Ischemic symptoms have been present for 2 years and the symptoms seemed to be aggravating and frequent during the preceding month. Physical examination showed the patient was conscious and found to have mild left upper extremity weakness and acalculia. MRI showed the presence of a diffusely dilated extensive vascular lesion mainly located in the corpus callosum, right frontal and temporal lobes intermingled with brain parenchyma without a clearly identifiable margin (Figure 1). MRA and MRV revealed a diffuse network of vessels in right frontal and temporal lobes with dilated arteries and drainage veins (Figure 2). Digital subtraction angiography
(DSA) revealed a large hypervascular lesion with a diffuse arterial supply involving the right anterior cerebral artery, right middle cerebral artery (MCA) and right posterior cerebral artery (PCA) without early venous filling, flow-related aneurysms, and main arterial stenosis were not observed, which is compatible with the findings for CPA (Figures 3 and 4). Considering the ischemic symptoms, we arrange the patient

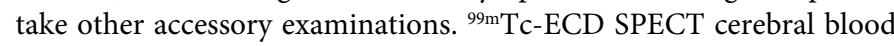
flow perfusion image revealed decreased perfusion in right frontal and parietal lobe with some segmental perfusion defect (Figure 5). PWMRI demonstrated increased relative cerebral blood flow (rCBF) and volume (rCBV), prolonged mean transit time (MTT), and decreased time to peak (TTP) at the lesion (Figure 6). The elevation signal on the CBF and CBV maps coincides with where vascular dilatation and proliferation were intense.

We performed right encephaloduromyosynangiosis (EDMS) to this patient. During the operation, we chose right extensive modified

*Corresponding author: Bin Xu, Department of Neurosurgery, Huashan Hospital, Fudan University, No.12 Wulumuqi Middle Road, 200040, Shanghai, PR China, Tel: 0086-21-52889999; E-mail: xubinww@yahoo.com

Received August 05, 2016; Accepted September 26, 2016; Published September 30, 2016

Citation: Li J, Gao X, Zhou Q, Gao C, Liao Y, et al. (2016) Encephaloduromyosynangiosis (EDMS) on a Pediatric Patient with Cerebral Proliferative Angiopathy. J Neurol Neurophysiol 7: 394. doi:10.4172/21559562.1000394

Copyright: (c) $2016 \mathrm{Li} \mathrm{J}$, et al. This is an open-access article distributed under the terms of the Creative Commons Attribution License, which permits unrestricted use, distribution, and reproduction in any medium, provided the original author and source are credited. 
Citation: Li J, Gao X, Zhou Q, Gao C, Liao Y, et al. (2016) Encephaloduromyosynangiosis (EDMS) on a Pediatric Patient with Cerebral Proliferative Angiopathy. J Neurol Neurophysiol 7: 394. doi:10.4172/2155-9562.1000394

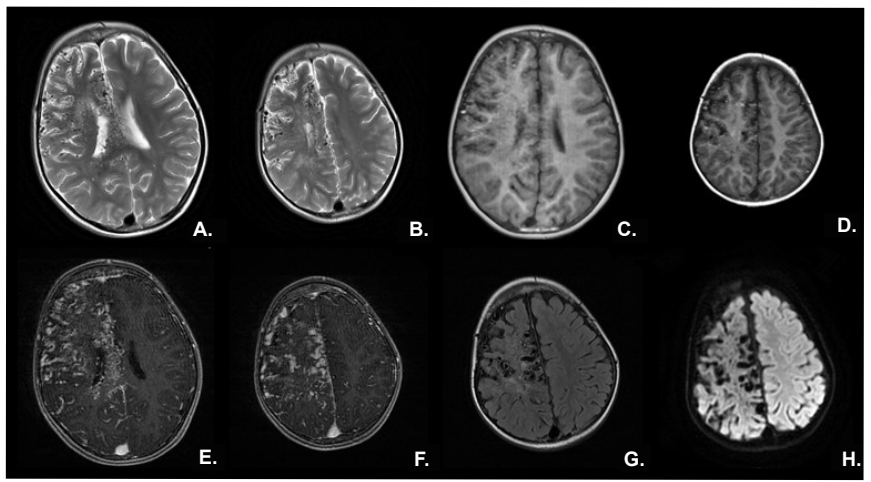

Figure 1: A-B T2 WI, C-D T1WI, G T2-FLAIR: Showed a diffusely dilated extensive vascular lesion which extended to right frontal, temporal lobes and corpus callosum. E-F: Enhanced MR showed the lesion can be enhanced significantly. H: DWI showed no apparent ischemic signal.

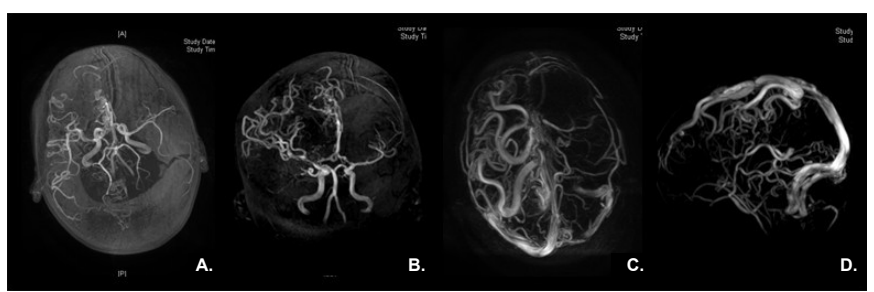

Figure 2: A-B MRA, C-D MRV showed a diffuse network of vessels with dilated arteries and drainage veins.
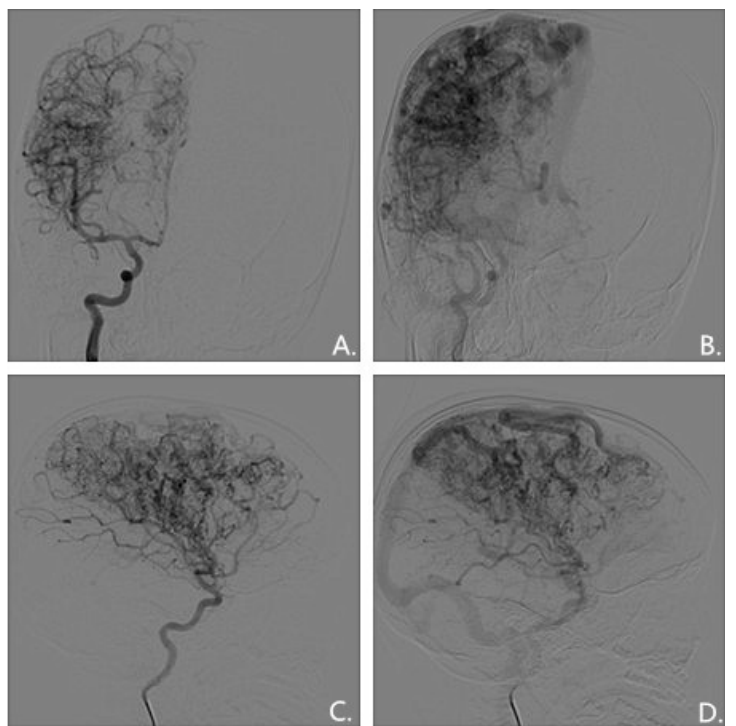

Figure 3: Digital subtraction angiography (DSA): Right internal carotid artery.

pterional approach combined with left coronal approach, and we found extensive dilated vessels passed through the brain surface after dura mater flip-over. The temporal muscle was placed on the pial surface of the brain, and the margins of temporal muscle was sutured with the Dura mater at the fold of the flip-over and fixed to the bone window.

The patient recovered well during the perioperative period, and no complication occurred. The patient exhibited disappearance of TIAs during the follow-up period. DSA at follow-up period revealed well compensation by profound neovascularization from ECA. ${ }^{99 \mathrm{~m} T c-}$ ECD SPECT obtained 6 months after operation showed significant improvement of right frontal and parietal hypoperfusion (Figure 5). MR perfusion at follow-up period demonstrated the range and blood flow of vascular lesion decreased significantly compared with preoperative MR perfusion.

\section{Discussion}

Cerebral proliferative angiopathy (CPA) is a rare cerebral vascular disease, which is different from typical AVM and characterized by diffuse vascular abnormalities with intermingled normal brain parenchyma [1]. Cerebral proliferative angiopathy has a complex clinical presentation. Clinical symptoms of CPA are seizures $(45 \%$ patients), headaches (41\%), focal neurological deficits (FNDs) not because of hemorrhage (16\%), and cerebral hemorrhages (12\%). Although it is rare that the onset of CPA is hemorrhagic, the chance of re-bleeding may be higher than in patients with typical AVMs $[1,2]$.

\section{Hemodynamic characteristics}

Preoperative ${ }^{99 \mathrm{~m}} \mathrm{Tc}-\mathrm{ECD}$ SPECT revealed significantly regional
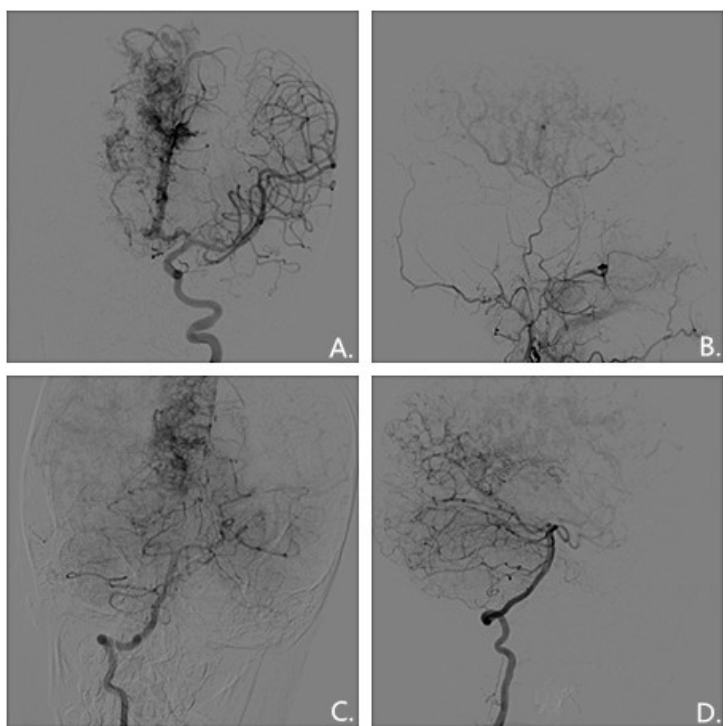

Figure 4: Digital subtraction angiography (DSA): A. Left internal carotid artery, B. Right external carotid artery, C-D. Right vertebral artery.

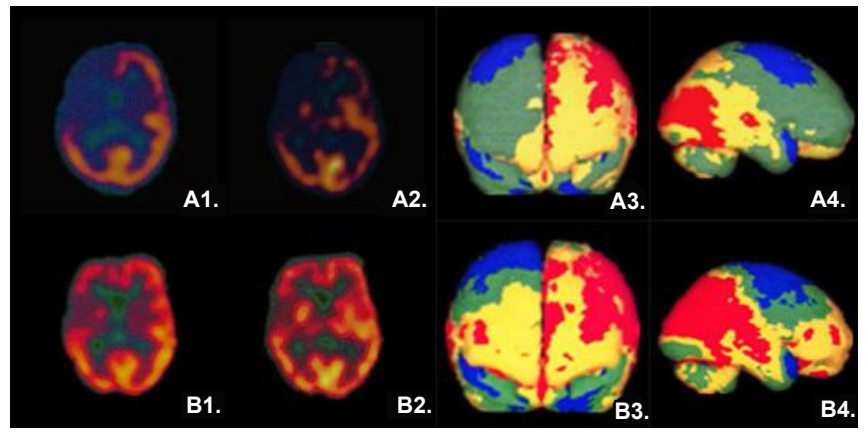

Figure 5: ${ }^{99 m}$ TC-ECD SPECT: A1-A4 (2015.11 Preoperative image) showed decreased perfusion in right frontal and parietal lobe with some segmental perfusion defect, B1-B4 (2016.05 follow-up) showed significant improvement of right frontal and parietal hypoperfusion. 


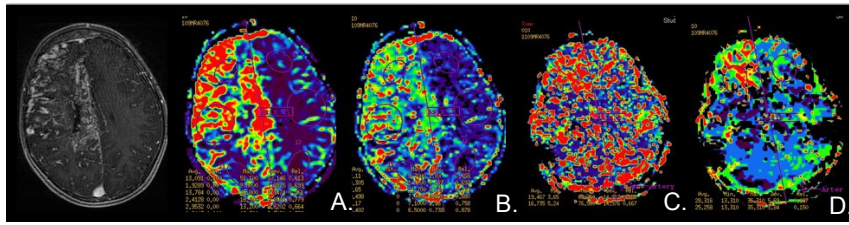

Figure 6: A rCBF, B rCBV, C MTT, D TTP PWI-MR (2015.11) showed increased relative cerebral blood flow (rCBF) and volume (rCBV), prolonged mean transit time (MTT), and decreased time to peak (TTP) at the lesion. The elevation signal on the CBF and CBV maps coincides with where vascular dilatation and proliferation were intense.

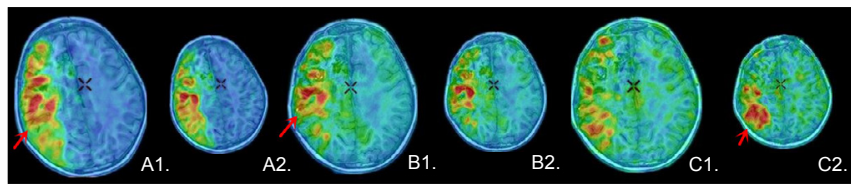

Figure 7: Territorial-ASL showed the range and blood flow of vascular lesion decreased significantly and well compensation by profound neovascularization from ECA. A1-A2 (2015.11): Preoperative perfusion region of right ICA. B1B2 (2016.05): Perfusion region of right ICA at follow-up. C1-C2 (2016.05): Perfusion region of right ECA at follow-up.

decreased perfusion in the area adjacent to the lesion. Kimiwada et al. [3] demonstrated severely impaired CVR over the affected hemisphere with CPA by acetazolamide stressed ${ }^{123}$ I-IMP SPECT. These studies all inferred that CPA was associated with the cerebral ischemia. Cerebral ischemia might occur at typical AVM by mechanism of "arterial steal phenomenon" due to massive arteriovenous shunts [4]. Perfusion pressure adjacent to the lesion decreased because of lowresistance arteriovenous fistulas, and $\mathrm{rCBF}$ in surrounding normal tissue decreased either. The arteriovenous shunts of CPA could not be detected in angiography. Nevertheless, CPA had similar pathological characteristics with AVM, and some study revealed massive microvascular arteriovenous shunts existed in CPA [1]. Therefore, cerebral ischemia in CPA still might be caused by "steal phenomenon", and abnormal vascular network proliferated because of ischemia.

Unlike SPECT cerebral blood flow perfusion image, CPA could presented increased relative cerebral blood flow ( $\mathrm{rCBF}$ ) and volume ( $\mathrm{rCBV}$ ), prolonged mean transit time (MTT), and decreased time to peak (TTP) at the lesion. SPECT use radiotracer such as ${ }^{99 \mathrm{~m}} \mathrm{Tc}-\mathrm{ECD}$ passively cross the blood-brain barrier to reflect cerebral perfusion, while PWI-MR is more sensitive to cerebral blood flow signal in the vascular bed. CPA is characterized with extensive diffuse vascular network, so relative cerebral blood flow (rCBF) and volume (rCBV) increase significantly in PWI-MR [5]. Furthermore, the elevation signal of $\mathrm{CBF}$ and $\mathrm{CBV}$ coincides with where vascular dilatation and proliferation were intense. However, CPA presents prolonged mean transit time (MTT), and decreased time to peak (TTP) at the lesion different from typical AVM in PWI-MR, which is more likely to hemodynamic characteristics of moyamoya disease (Figure 7).

\section{Treatment}

There is no consensus on treatment of cerebral proliferative angiopathy at present. Previous case reports proposed partial embolization of abnormal vascular lesion, and bevacizumab was used for treatment because of elevated level of VEGF in the patient's CSF [6]. However, the patient's ischemic symptoms were not relieved at followup. Considering it is rare that the initial onset of CPA is hemorrhagic, interventional embolization is not advocated because normal brain tissue might be embolized [1]. Previous studies did not demonstrate the relationship between the proliferation of abnormal vascular network and the hypoperfusion with cerebral ischemia in the patients with CPA, but the hemodynamic characteristics of CPA were more similar to moyamoya disease in the present PWI-MR study. Therefore, we speculated that cerebral ischemia in CPA might be caused by "steal phenomenon" and some other mechanisms, and cerebral ischemia might stimulate the proliferation of abnormal capillary network simultaneously. Furthermore, increased regional cerebral blood flow perfusion may relieve clinical symptoms and improve prognosis of patient with CPA. Surgical procedures for treating moyamoya disease which can increase regional cortex's cerebral blood flow perfusion may benefit patients with CPA, Kono et al. [2]. reported a case of adult CPA with cerebral ischemia in which neurological deficits were improved after encephaloduroarteriosynangiosis (EDAS). Encephaloduromyosynangiosis (EDMS) can enable the patients to take full advantage of blood supply from the external carotid artery in a wider area, according to our previous experience in treating moyamoya disease [7]. Therefore, we chose surgical procedure of encephaloduromyosynangiosis (EDMS) to treat this pediatric CPA patient with ischemic symptoms. The patient's symptoms have been relieved significantly after the operation, and the patient exhibited disappearance of TIAs during the follow-up period. ${ }^{99 \mathrm{~m}} \mathrm{Tc}$-ECD SPECT obtained 6 months after operation showed significant improvement of right frontal and parietal blood perfusion. MR perfusion at followup period demonstrated the range and blood flow of vascular lesion decreased significantly compared with preoperative MR perfusion. Follow-up angiography studies showed well compensation by profound neovascularization through the superficial temporal artery,

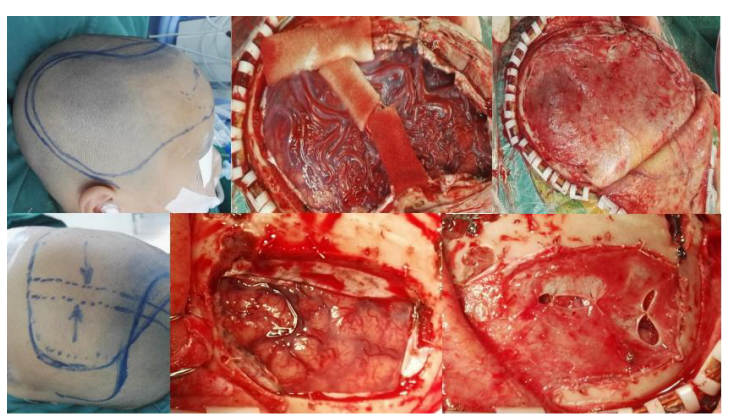

Figure 8: Intraoperative images of encephaloduromyosynangiosis (EDMS): Extensive dilated vessels are present on the surface of the brain.

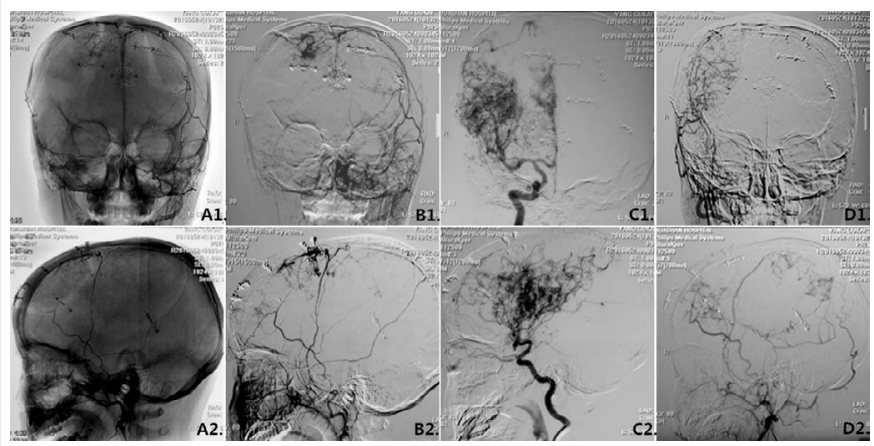

Figure 9: DSA at follow-up (2016.05) showed well compensation by profound neovascularization through the superficial temporal artery, and vascular malformation disappeared around the region supplied from the ECA. (B1-2 Left ECA, C1-2 Right ICA, D1-2 Right ECA). 
Citation: Li J, Gao X, Zhou Q, Gao C, Liao Y, et al. (2016) Encephaloduromyosynangiosis (EDMS) on a Pediatric Patient with Cerebral Proliferative Angiopathy. J Neurol Neurophysiol 7: 394. doi:10.4172/2155-9562.1000394

Page 4 of 4

and vascular malformation disappeared around the region supplied from the ECA. EDMS has been effective with fair prognosis and low rate of perioperative complication in pediatric patients. Therefore, EDMS procedure may be a reasonable and effective treatment for patients with ischemia associated with CPA (Figures 8 and 9).

\section{References}

1. Lasjaunias PL, Landrieu P, Rodesch G, Alvarez H, Ozanne A, et al. (2008) Cerebral proliferative angiopathy: Clinical and angiographic description of an entity different from cerebral AVMs. Stroke 39: 878-885.

2. Kono K, Terada $T$ (2014) Encephaloduroarteriosynangiosis for cerebra proliferative angiopathy with cerebral ischemia. J Neurosurg 121: 1411-1415.

3. Kimiwada T, Hayashi T, Shirane R, Tominaga T (2013) 123I-IMP-SPECT in a patient with cerebral proliferative angiopathy: A case report. J Stroke Cerebrovasc Dis 22: 1432-1435.

4. Kim DJ, Krings $T$ (2011) Whole-brain perfusion CT patterns of brain arteriovenous malformations: A pilot study in 18 patients. AJNR Am J Neuroradiol 32: 2061-2066.

5. Vargas MC, Castillo M (2011) Magnetic resonance perfusion imaging in proliferative cerebral angiopathy. J Comput Assist Tomogr 35: 33-38.

6. Marks MP, Steinberg GK (2012) Cerebral proliferative angiopathy. J Neurointerv Surg 4: e25.

7. Xu B, Song DL, Mao Y, Gu $Y X, X u H$, et al. (2012) Superficial temporal artery-middle cerebral artery by-pass combined with encephalo-duro-myosynangiosis in treating moyamoya disease: surgical techniques, indications and midterm follow-up results. Chin Med J (Engl) 125: 4398-4405. 